\title{
Reflets
}

Revue ontaroise d'intervention sociale et communautaire

\section{Entre le marteau et l'enclume : le vécu des intervenantes et intervenants interculturels}

\section{Mathilde Nduwimana}

Volume 4, numéro 1, printemps 1998

Intervention en contextes minoritaires

URI : https://id.erudit.org/iderudit/026204ar

DOI : https://doi.org/10.7202/026204ar

Aller au sommaire du numéro

Éditeur(s)

Reflets : Revue ontaroise d'intervention sociale et communautaire

ISSN

1203-4576 (imprimé)

1712-8498 (numérique)

Découvrir la revue

Citer cet article

Nduwimana, M. (1998). Entre le marteau et l'enclume : le vécu des intervenantes et intervenants interculturels. Reflets, 4(1), 176-181. https://doi.org/10.7202/026204ar

Tous droits réservés (C) Reflets : Revue ontaroise d'intervention sociale et communautaire, 1998
Ce document est protégé par la loi sur le droit d'auteur. L'utilisation des services d'Érudit (y compris la reproduction) est assujettie à sa politique d'utilisation que vous pouvez consulter en ligne.

https://apropos.erudit.org/fr/usagers/politique-dutilisation/ 


\title{
Entre le marteau et l'enclume:
}

\section{le vécu des intervenantes et intervenants interculturels}

\author{
Mathilde Nduwimana \\ Travailleuse sociale, Ottawa
}

Le Canada accueille depuis des années de nouvelles personnes en provenance de divers pays du monde. Les services sociaux et de santé disponibles pour aider ces personnes à s'adapter et à s'intégrer cheminent vers l'adaptation de leurs approches d'intervention, afin de mieux servir cette clientèle. En effet, de plus en plus d'organismes socio-communautaires s'efforcent de répondre aux besoins particuliers des clients issus des communautés ethnoculturelles, en se dotant des outils nécessaires à cet effet. On remarque notamment que ces organismes embauchent des intervenantes et des intervenants issus des communautés ciblées, afin de pouvoir tenir compte des obstacles ayant trait à l'accès aux services, comme les barrières d'ordre culturel.

Dans cet article, je me propose de présenter des observations et des réflexions personnelles réalisées au cours de quelques années de pratique comme intervenante interculturelle au sein des organismes communautaires offrant des services sociaux aux personnes immigrantes de la Municipalité régionale d'OttawaCarleton.

Grâce à mon expérience en intervention interculturelle, j'ai pu observer certains défis liés à la pratique interculturelle. En effet, dans la pratique quotidienne, je me suis rendu compte que les intervenantes ou intervenants issus des communautés ethnoculturelles 
peuvent avoir de la difficulté à concilier deux types d'attentes: celles des responsables des organismes pourvoyeurs de services sociaux et de santé, et celles de la clientèle immigrante ou réfugiée.

\section{Des attentes contradictoires}

Dans la majorité des cas, les responsables des organismes pourvoyeurs des services s'attendent à ce que les usagères ou les usagers immigrants s'adaptent rapidement au mode de fonctionnement du pays d'accueil. Ils veulent en fait une intégration rapide et complète de ces personnes. De son côté, la clientèle immigrante ou réfugiée s'attend à ce que les intervenantes ou intervenants issus de leurs communautés les aident à surmonter rapidement les difficultés d'accès aux ressources et services. En considérant les attentes des responsables des organismes pourvoyeurs des services, j'ai réalisé qu'elles sont en partie liées au fait que ces derniers ne sont pas très bien informés ni des réalités culturelles des personnes immigrantes ou réfugiées, ni de leur processus d'adaptation. Une intégration rapide et complète suppose un changement radical par rapport au mode de fonctionnement habituel des usagères et des usagers ciblés ou alors, une autre perception du monde nouveau qui les entoure. On remarque cependant que le processus d'adaptation au mode de fonctionnement du pays d'accueil n'est pas uniforme et qu'il peut varier en fonction de plusieurs facteurs. Comme le souligne Abou (1988:5), les jeunes immigrants s'adaptent facilement et s'ajustent rapidement au mode de fonctionnement du pays d'accueil. Cela est moins le cas pour les personnes immigrantes adultes. En effet, comme le souligne aussi Abou, le noyau dur de l'identité culturelle d'une personne résiste au changement ou à l'adaptation à une autre culture. Le noyau dur réfere ici à la mentalité ou aux manières de penser et de sentir qui n'ont pas été affectées par la culture du pays d'accueil. En effet, certaines valeurs, surtout celles de type émotif, peuvent longtemps résister au changement. Les élément périphériques peuvent subir une acculturation aux normes de la 
société d'accueil, suite à la nécessité, par exemple, de s'intégrer au marché du travail. Ces personnes vont par exemple apprendre une ou les deux langues officielles, pour pouvoir mieux intégrer le marché de l'emploi, laissant intact le noyau dur de la culture du pays d'origine. On peut alors comprendre combien il est difficile pour les personnes immigrantes de s'ajuster aux codes de fonctionnement du pays d'accueil, puisqu'elles ont intégré tout au long de leur socialisation des façons de fonctionner différentes de celles des membres de leur société d'accueil.

Les intervenantes ou intervenants issus des communautés ethnoculturelles sont dans ces conditions tiraillés entre les attentes, les valeurs et les demandes des deux cultures, à savoir celles des organismes traditionnels, et celles des personnes immigrantes avec lesquelles ils sont appelés à travailler. Les premiers s'attendent à ce que les intervenantes ou intervenants interculturels contribuent à l'adaptation rapide de la clientèle immigrante ou réfugiée au mode de fonctionnement des organismes. Tel est le cas de la perception du temps: la ponctualité, par exemple, est une valeur très importante au niveau du fonctionnement dans le monde occidental. Dans la plupart des pays d'origine de la clientèle ciblée, c'est l'objectif à atteindre qui importe le plus. Les gens vont plus se soucier d'atteindre un objectif fixé, plutôt que de donner de l'importance au temps qu'il faut prendre pour atteindre cet objectif. La rencontre de deux façons de percevoir le temps occasionne souvent de l'incompréhension entre les actrices et/ ou les acteurs impliqués.

Du côté de la clientèle ciblée, la plupart des personnes s'attendent à ce que les intervenantes ou intervenants issus de leurs communautés soient en mesure de les aider à surmonter les multiples obstacles d'accès aux services et aux ressources disponibles dans la communauté d'accueil. C'est, par exemple, le cas au niveau des services de recherche d'emploi où la plupart des clientes ou clients viennent avec l'espoir que les intervenantes ou intervenants vont les aider à obtenir rapidement un emploi. Ces attentes sont en fait liées au fait que, dans de nombreux pays d'origine des personnes immigrantes ou réfugiées, les gens n'ont pas à se chercher d'emploi, puisqu'ils sont nommés à des postes, 
une fois les études terminées. Il n'est pas toujours aisé de trouver un juste équilibre relativement à la conciliation de ces différentes attentes. Cette situation peut facilement compromettre la confiance de la clientèle envers l'efficacité des services et envers les intervenantes ou intervenants qui peuvent être perçus comme étant très peu utiles.

\section{Des projets trop ponctuels}

Des difficultés sont aussi souvent observées quant au développement de la confiance envers des pourvoyeurs de services. En effet, dans la majorité des cas, les projets développés à l'intention des personnes immigrantes sont ponctuels. Cela a comme impact de limiter le niveau de confiance et d'implication de ces personnes, qui jugent inutile de collaborer à des projets qui ne vont pas durer longtemps, malgré la persistance des besoins. Ainsi, non seulement les intervenantes et intervenants interculturels éprouvent de la difficulté à maitriser des approches d'intervention interculturelle, puisqu'elles ou ils ont peu de temps pour en appliquer les théories et pour nourrir ces dernières d'expériences vécues sur le terrain, mais aussi il leur est difficile de recruter les participants aux projets. Cette peur de vivre un perpétuel recommencement s'accompagne de la difficulté de pouvoir mesurer l'impact ou les résultats à moyen et à long terme des interventions réalisées auprès de la population ciblée. En effet, la façon dont les projets d'adaptation et d'intégration des personnes immigrantes ou réfugiées fonctionnent suscite un questionnement : peut-on et doit-on juger du succès des programmes par le nombre de participants aux activités, ou par le développement de réseaux socio-communautaires beaucoup plus fluides et moins quantifiables?

En conclusion, j'estime qu'un travail continu de sensibilisation des pourvoyeurs des services sur le processus d'adaptationintégration des personnes immigrantes et des réfugiés doit se poursuivre. Cela permettrait de briser les stéréotypes entretenus 
d'un côté comme de l'autre. Les pourvoyeurs des services pourraient avoir l'occasion d'être mieux informés sur les réalités reçues par la clientèle dans la société d'accueil. La clientèle immigrante pourrait, de son côté, être sensibilisée aux codes et aux normes de fonctionnement de cette société d'accueil.

Au niveau du développement des projets destinés à faciliter le processus d'adaptation-intégration des personnes immigrantes et des réfugiées, il s'avère indispensable de penser à des projets qui permettraient d'évaluer les résultats. Dans le même ordre d'idées, ces projets devraient être ceux qui favorisent le développement d'un esprit d'appartenance à la société d'accueil, chez les bénéficiaires, en leur permettant de se rendre compte de l'intérêt qu'il y a à participer aux projets, parce qu'on y retire des avantages. Je pense notamment aux projets qui, sur une base progressive, permettent aux différents membres de la communauté de se rapprocher, de se connaitre et se reconnaitre, et finalement de développer une cohabitation plus harmonieuse.

De plus, cette manière d'agir permettrait aux personnes intervenant auprès de cette clientèle de maitriser les outils d'intervention, parce qu'elles auraient le temps d'appliquer des approches d'intervention spécifiques, de les adapter ou de les améliorer au cours de leur pratique. J'estime également que les personnes qui interviennent auprès de la clientèle immigrante et qui n'ont pas reçu de formation interculturelle pourraient améliorer leur pratique si dans le cadre des projets développés pour faciliter l'adaptation-intégration des personnes immigrantes ou des réfugiées, on ajoutait un volet de formation continue.

Dans le même ordre d'idée, j'estime que pour remédier à cette situation, les personnes intervenantes issues des communautés immigrantes devraient s'organiser pour créer un espace de réflexion autour des questions qui surgissent au cours de leur pratique quotidienne et cela dans une perspective de développement professionnel. On pourrait, suite aux idées qui sortiraient de ces rencontres, penser à un cadre beaucoup plus structuré, comme un groupe de réflexion et de développement des approches d'intervention adaptées à la réalité interculturelle, telle qu'observée dans les divers milieux d'intervention socio- 
communautaire. À mon avis, cela leur permettrait, d'échanger des expériences, de bénéficier d'un soutien mutuel, de réfléchir aux ressources nécessaires pour améliorer l'intervention et aux stratégies qui leur permettraient d'avoir accès à ces ressources. 